\title{
Microorganismes entomopathogènes, prédateurs et parasites des moustiques: Perspectives pour la lutte raisonnée contre les vecteurs du paludisme en Afrique sub-saharienne
}

\author{
A.Z. ABAGLI ${ }^{1}$, T.B.C. ALAVO ${ }^{1 *}$ et J. BRODEUR ${ }^{2}$ \\ ${ }^{I}$ Laboratoire d'Entomologie Appliquée, Faculté des Sciences et Techniques, Université d'Abomey-Calavi, \\ BP 215 Godomey, Bénin. \\ ${ }^{2}$ Département des Sciences Biologiques, Université de Montréal, Canada. \\ *Auteur correspondant ; E-mail : thieryalavo@ hotmail.com
}

\section{RÉSUMÉ}

En Afrique sub-saharienne, les moustiques sont des vecteurs de plusieurs maladies dont le paludisme. Pour lutter contre les moustiques, les insecticides de synthèse sont intensivement utilisés soit pour la pulvérisation des gîtes larvaires et des domiciles, soit pour l'imprégnation des moustiquaires. Cette utilisation intensive d'insecticides chimiques a conduit au développement du phénomène de résistance chez les moustiques; ceci rend de plus en plus difficile la lutte contre ces insectes. Dans la nature, un ensemble d'ennemis naturels contribuent à la régulation des populations de moustiques. Certains de ces organismes sont produits et utilisés à grande échelle dans les pays industrialisés pour la lutte raisonnée contre les moustiques. Cet article fait le point des connaissances actuelles des ennemis naturels des moustiques en vue de mettre en exergue le rôle que pourraient jouer ces organismes utiles pour la gestion intégrée des vecteurs du paludisme en Afrique sub-saharienne. Il ressort de la présente synthèse bibliographique que les bactéries Bacillus thuringiensis var. israelensis et Bacillus sphaericus sont les plus utilisées pour lutter contre les larves de moustiques. Le nématode entomopathogène, Romanomermis iyengari, très efficace contre les larves d'anophèle revêt un intérêt particulier pour l'Afrique du fait de la possibilité de le produire à grande échelle avec des moyens simples. Le poisson larvivore Gambusia affinis peut également contribuer à la gestion des populations de moustiques, notamment dans les zones de productions aquacoles.

(C) 2014 International Formulae Group. All rights reserved.

Mots clés : Paludisme, moustiques, vecteurs, ennemis naturels, lutte anti-vectorielle

\section{INTRODUCTION}

Selon l'Organisation Mondiale de la Santé (OMS), les estimations en 2010 font état de 216 millions d'épisodes palustres, dont $81 \%$ dans la région Afrique, soit 174 millions de cas. Le nombre des décès dus au paludisme est estimé pour l'année 2010 à 655 000, dont $91 \%$ en Afrique. A l'échelle mondiale, $86 \%$ des décès imputables au paludisme ont frappé des enfants de moins de 5 ans (OMS, 2011).

Pour lutter contre les vecteurs du paludisme, des insecticides de synthèse sont intensivement utilisés soit pour la pulvérisation des gîtes larvaires et des domiciles, soit pour l'imprégnation des 
moustiquaires et des rideaux. Cette utilisation intensive d'insecticides chimiques a conduit au développement du phénomène de résistance (Djogbénou et al., 2011). Cette résistance se manifeste même pour l'organochloré DDT, pourtant réputé pour son effet choc et son extrême rémanence (Balkew et al., 2006). Ceci rend de plus en plus difficile la lutte contre les moustiques. À ce problème de résistance, s'ajoute le rôle néfaste des pesticides chimiques sur la santé humaine et l'environnement. S'il est vrai que la toxicité immédiate de la majorité des produits utilisés est généralement faible, l'utilisation de ces produits chimiques peut, à long terme, nuire gravement à la santé publique, plus particulièrement en matière de fécondité et de cancer (de Jager et al., 2012 ; Carson, 2002).

Face à ces problèmes, il est urgent d'envisager l'utilisation de moyens alternatifs pour la lutte antivectorielle. Dans cette optique, les ennemis naturels des moustiques peuvent être considérés.

Cet article est une revue de la littérature internationale relative aux travaux qui portent sur les microorganismes pathogènes et les prédateurs des moustiques. Il met en exergue le rôle que pourraient jouer ces organismes en matière de lutte raisonnée contre les vecteurs du paludisme en Afrique sub-saharienne.

\section{MICROORGANISMES ENTOMOPATHOGÈNES}

\section{Les champignons}

La susceptibilité des larves de moustiques aux champignons pathogènes appartenant aux genres tels que Lagenidium, Coelomomyces, Culicinomyces, Beauveria, Metarhizium et autres a été largement étudiée (Scholte et al., 2004; Luz et al., 2007). Kerwin (2007) a testé le champigon Lagenidium giganteum (Oomycètes) sur les larves de moustique et a constaté que les larves infectées meurent entre 24 h et $60 \mathrm{~h}$ et les champignons sont capables de se recycler dans l'environnement pendant des mois, voire des années.

Les espèces comme Beauveria bassiana et Metarhizium anisopliae se sont révélées efficaces contre les larves et les adultes des espèces de moustiques comme Aedes aegypti, Ae. albopictus, Culex quinquefasciatus, $C x$. pipiens, $C x$. tarsalis, Cx. tritaeniorhynchus, An. stephensi et An. albimanus au laboratoire et en milieu naturel (Clark et al., 1968; Alves et al., 2002; Wright et al., 2005 ; Scholte et al., 2007 ; Kanzok et Lorena, 2006 ; Luz et al., 2007 ; Suman et al., 2008; Siva et Kadarkarai, 2011).

D'autres études ont également montré que les larves de moustiques sont susceptibles aux infections par les champignons tels que Leptolegna chapmanii (Lord et Fukuda, 1988), Aspergillus parasiticus (Hati, 1965), Aspergillus spp. (Moraes et al., 2001), Penicillium spp. (Mvoutoulou, 1992 ; Costa et al., 1998), Tolypocladium spp. (Soares et al., 1985 ; Goettel, 1988), Aspergillus flavus, Aspergillus parasiticus, Penicillium falicum, Fusarium vasinfectum et Trichoderma viride (Govindarajan et al., 2005), Aspergillus clavatus (Seye et al., 2009).

Par ailleurs, Russell et al. (2001) ont conclu après des travaux réalisés en Australie que le champignon Penicillium citrinum Thom a contribué à réduire la viabilité des œufs d'Ae. Aegypti. Albernaz et al. (2009) quant à eux, ont montré que $M$. anisopliae a une activité ovicide sur les œufs d'Ae. Aegypti. Luz et al. (2007) ont étudié en laboratoire, l'effet de plusieurs autres champignons sur les œufs du moustique $A e$. aegypti. Ces auteurs ont trouvé que $B$. bassiana, Evlachovaea kintrischica, $M$. anisopliae, Paecilomyces carneus, Isaria farinosa, I. fumosorosea, P. marquandii, P. lilacinus, et Penicillium sp. ont eu une forte activité ovicide contre Ae. aegypti.

En gros, une forte humidité relative de l'air est la condition nécessaire pour le 
développement des champignons sur les œufs d'Ae. Aegypti (Santos et al., 2009).

\section{Les bactéries}

Bacillus thuringiensis var. israelensis et Bacillus sphaericus sont les bactéries les plus connues et les plus utilisées pour lutter contre les larves de moustiques. Depuis leur identification, les spécificités de leur microbiologie, génétique, biologie moléculaire, ainsi que leur activité et efficacité contre les organismes cibles ont été largement étudiées dans plusieurs pays du monde (Su et Mulla, 1999; Zahiri et al., 2002 ; Monnerat et al., 2004 ; Armengol et al., 2006 ; Lacey, 2007 ; Silva-Filha et al., 2008 ; Otieno-Ayayo et al., 2008 ; Rout et al., 2011). De même, leur effet sur l'environnement et sur les organismes noncibles a été bien évalué et les résultats ont montré que ces agents de lutte biologique ont une efficacité élevée, et sont inoffensifs pour l'environnement et beaucoup d'organismes non cibles (Roberts, 1995).

Par ailleurs, d'autres espèces de bactéries ont montré une activité insecticide contre les larves et les pupes de moustiques ; il s'agit par exemple de Brevibacillus laterosporus (Ruiu et al., 2007) et de Bacillus subtilis subsp. subtilis (Geetha et Manonmani, 2007). Enfin, les métabolites de bactéries comme Pseudomonas fluorescens, $P$. pseudomallei et $P$. aeruginosa se sont révélés toxiques aux pupes de moustiques tels que $C x$. quinquefasciatus, An. stephensi et Ae. aegypti (Prabakaran et Hoti, 2008 ; Prabakaran et al., 2009).

Diverses méthodes de production et de formulation des bactéries entomopathogènes ont été explorées en vue d'améliorer leur coût et leur qualité (Xudong et al., 1993; Promdonkoy et al., 2004 ; Ohgushi et al., 2005 ; Park et al., 2005; Prabakaran et Balaraman, 2006; Ramirez-Suero et al., 2005 ; Sarrafzadeh et al., 2005 ; Federici et al., 2007 ; Prabakaran et al., 2007, 2009 ; Poopathi et Abidha, 2009).
Néanmoins, des problèmes de résistance vis-à-vis de ces biopesticides ont été déjà signalés (Zahiri et al., 2002 ; SilvaFilha et al., 2008 ; Olivera et al., 2009).

\section{Les virus}

Le premier virus pathogène des moustiques a été découvert en 1963; il s'agit d'un densovirus putatif de Culex tarsalis qui a été identifié en Californie (Kellen et al., 1963).

D'autres virus pathogènes de moustiques ont été également signalés dans d'autres régions du monde (Federici, 1985). Ce sont :

- Les baculovirus (NPVs) (Baculoviridae) qui causent la polyédrose nucléaire chez les moustiques. Il s'agit entre autre de CuniNPV et UrsaNPV qui sont respectivement responsables de la polyédrose nucléaire chez les moustiques $C x$. nigripalpus et Uranotaenia sapphirina (Federici, 1985 ; Becnel et al., 2001 ; Andreadis et al., 2003 ; Shapiro et al., 2004).

- Les cypovirus (CPVs) (Reoviridae) qui sont responsables de la polyédrose cytoplasmique. Il s'agit par exemple de CrCPV qui cause la polyédrose cytoplamique chez les moustiques $C x$. restuans et de UsCPV, agent pathogène de la même maladie chez le moustique Ur. sapphirina (Becnel et White, 2007).

- Les densovirus (DNVs)

(Parvoviridae). Le densovirus rencontré chez Ae. albopictus s'appelle AalDNV, et celui rencontré chez $C x$. pipiens est CpDNV (Ledermann et al., 2004 ; Büchen-Osmond, 2003 ; Jousset et al., 2000).

- Les iridovirus (MIVs) (Iridoviridae) qui sont aussi des virus pathogènes des moustiques.

Les baculovirus, densovirus et iridovirus sont des virus dont le matériel génétique est formé d'ADN, tandis que les cypovirus sont les principaux virus à matériel 
génétique formé d'ARN (Becnel et White, 2007).

\section{Prédateurs}

Poissons larvivores

Dans différentes régions du monde, des poissons indigènes ont été utilisés pour contrôler les larves de moustiques (Morton et al., 1988 ; Neng et al., 1987 ; Wu et al., 1991; Yu, 1986; Yu et Kim, 1993; Kim et al., 1994; Martinez-Ibarra et al., 2002; Hurst et al., 2006 ; Marti et al., 2006).

Gambusia affinis, Poecilia reticulata, Oreochromis mossambicus, O. niloticus, Aplocheilus dayi, Laubuca Chela, Esomus thermoicos, Puntius filamentosus, Rasbora daniconius, Anabas testudineus, Clarias batrachus, Heteropneustes fossilis, Tilapia guineensis, Epiplatys spilargyreius, Tilapia zilll et Melanotaenia duboulayi ont été reconnues comme des espèces de poissons larvivores (Schleier et al., 2008; Asimeng et Mutinga, 1992 ; Valero et al., 2006 ; Walton, 2007 ; Hurst et al., 2007 ; Kusumawathie et al., 2006 ; Bhattacharjee et al., 2009 ; Louca et al., 2009).

De tous ces poissons, G. affinis est l'espèce la plus utilisée en matière de lutte biologique contre les larves de moustiques.

\section{Insectes entomophages}

- Diptères prédateurs

Dans l'ordre des diptères, il existe des espèces de moustiques qui sont prédateurs d'autres espèces de moustiques. Les moustiques prédateurs les plus connus sont les Toxorhynchites. Ce sont des moustiques dont les larves sont prédatrices de larves d'autres moustiques (Schreiber, 2007).

Les espèces d'Aedes du sous-genre Alanstonea, ainsi que celles des genres comme Lutzia, Ochlerotatus et Psorophora sont des prédateurs obligatoires. Quelques autres espèces de moustiques sont considérées comme prédatrices facultatives d'autres moustiques (Mogi, 2007).

Il existe de nombreuses autres espèces de diptères qui se nourrissent des larves ou adultes de moustiques. Il s'agit par exemple de: Mochlonyx cinctipes, Chaoborus americana, Corethrella appendiculata, Anatopynia pennipes et de Mycetophilides Xenoplatyura (Mogi, 2007).

\section{- Les hémiptères prédateurs}

La famille des Notonectidae est considérée comme la plus prometteuse en lutte biologique contre les moustiques. Les travaux réalisés au laboratoire et en milieu naturel ont montré que la présence de certaines espèces de cette famille a permis la réduction de la densité des larves et pupes de moustiques; il s'agit par exemple d'espèces prédatrices comme Notonectida hoffmani, $N$. undulata et $N$. kirbyi. Toutefois l'utilisation de ces entomophages dans la pratique serait compliquée parce que les quelques moustiques qui leur sont vulnérables évitent de pondre des œufs dans les eaux infestées de ces prédateurs (Quiroz-Martínez et Rodriguez-Castro, 2007 ; Mogi, 2007).

Il existe aussi des fourmis appelées Solenopsis invicta qui mangent ou endommagent les œufs terrestres d'Ae. albopictus et Psorophora columbiae au laboratoire et en champ expérimental (Lee et al., 1994 ; Burnham et al., 1994).

\section{- Coléoptères prédateurs}

On retrouve des espèces prédatrices de moustiques au sain de la famille des Dytiscidae et Hydrophilidae. L'activité de ces coléoptères prédateurs dans les étangs permanents et temporaires ainsi que dans les champs de riz était la cause d'une mortalité importante du moustique Culex tarsalis. Ces coléoptères attaquent les larves et pupes de moustiques (Mogi, 2007 ; Quiroz-Martínez et Rodriguez-Castro, 2007).

\section{- Odonates prédateurs}

Les espèces d'Odonates comme Sympetrum frequens, Pistia striates et Crocothemis servilia sont prédatrices des larves d'An. sinensis, de Mansonia spp. et d'Ae. aegypti (Mogi, 2007).

Certains moustiques ont évité de pondre leurs œufs dans des piscines expérimentales contenant les Odonates; ceci pourrait rendre difficile l'utilisation de ces 
prédateurs comme moyens de lutte biologique contre les moustiques (QuirozMartínez et Rodriguez-Castro, 2007 ; Mogi, 2007).

\section{Les arachnides}

Les araignées appartenant aux genres Dolomedes (Pisauridae), Pirata et Pardosa (Lycosidae) vivent à la surface de l'eau et attaquent les larves de moustiques comme Cx. pipiens, An. messeae et Cx. modestus.

Par ailleurs, certaines araignées comme Heteropoda venatoria (Sparassidae), Hygropoda dolomedes (Pisauridae), Crossopriza lyoni (Pholcidae), Salticus scenisus (Salticidae), et bien d'autres se nourrissent d'adultes de moustiques vecteurs de maladies.

En outre, certains acariens aquatiques (Hydrachnida) sont prédateurs des moustiques Ae. albopictus Ae. vittatus et Armigeres subalbatus (Mogi, 2007).

\section{Crustacés, Annélides et Plathelminthes prédateurs \\ Dans ce groupe d'invertébrés, les} copépodes cyclopoïdes sont les plus connus en matière de lutte biologique contre les larves de moustiques. Les copépodes cyclopoïdes sont de petits crustacés qui s'attaquent aux larves de moustiques du premier et deuxième stade. Les espèces comme Mesocyclops aspericornis, $M$. Aspericornis, M. Aspericornis, $M$. Aspericornis et $M$. longisetus sont reconnues comme prédatrirces des larves des moustiques Ae. aegypti, Cx. quinquefasciatus et An. farauti (Kay et al., 1992 ; Brown et al., 1991).

Par ailleurs, les têtards du crustacé californien Triops newberryi (Triopsidae: Nostraca) se nourrissent des larves de $C x$. quinquefasciatus et de $C x$. tarsalis. Ces moustiques pondent également moins d'œufs dans les gîtes contenant les têtards de crustacées. Aussi, la langouste rouge des marais de Louisiane, Procambarus clarkia, introduite en Afrique s'est avérée être prédatrice potentielle pour An. gambiae (Mogi, 2007).

Les annélides et les plathelminthes jouent également un rôle dans la régulation des populations de moustiques. Il existe une espèce d'annélide qui est reconnue comme étant prédatrice des moustiques. Il s'agit de la sangsue Helobdella triserialis qui se nourrit des larves d'Ae. fluviatilis et de $C x$. quinquefasciatus (Mogi, 2007).

Quant aux plathelminthes, il y a par exemple Dugesia tigrina qui tue les larves de moustiques par capture directe ou par piégeage dans un mucus qu'il secrète. Des essais ont montré que ce plathelminthe réduit de façon significative la densité des larves de Culex (Mogi, 2007). Il existe bien d'autres espèces de plathelminthes comme Girardia anceps qui contribue également à la régularisation des populations de moustiques (Tranchida et al., 2009).

\section{Nématodes mermithidés}

Les nématodes Mermithidae (ordre des Mermithida) sont généralement longs, minces et translucides (blanc); leur longueur varie de 10 à 100 millimètres. Ils sont parasites des arthropodes. Le système taxonomique actuel place l'ordre des Mermithida dans la classe des Enoplea (De Ley et Blaxter, 2002), où l'on rencontre la majorité des nématodes parasites des insectes.

La biologie des mermithidés dans les populations d'insectes aquatiques fait participer les juvéniles pré-parasitiques infectieux (juvéniles de $2^{\text {ème }}$ stade). Ces juvéniles pré-parasitiques infectieux pénètrent dans l'espèce hôte par le tégument. Par la suite, le juvénile parasite se développe, mue une fois, et après, stocke suffisamment de nourriture pour sa phase adulte. Le nématode tue l'hôte au moment de son émergence du corps de ce dernier en tant que juvénile post-parasitique (Platzer, 2007).

Le développement de la phase adulte et la reproduction ont lieu à l'extérieur de l'hôte mais toujours dans son environnement. 
Dans le cas des mermithidés parasites des moustiques, les juvéniles post-parasitiques se retrouvent dans le substrat du gîte. Le fait que les nématodes mermithidés tuent leur hôte a été un facteur déterminant pour l'exploitation de ces nématodes en lutte biologique.

Les travaux sur les nématodes mermithidés ont mis en évidence les avantages de ces vers ronds en matière de lutte biologique: facilité d'application, sécurité environnementale, spécificité vis-àvis des moustiques, facilité d'élevage en masse, et possible recyclage des nématodes dans l'environnement (Petersen, 1985; Platzer et al., 2005 ; Popiel et Hominick, 1992 ; Federici, 1995 ; Kerry et Hominick, 2002).

Les recherches ont également démontré que les mermithidés exercent effectivement des effets dévastateurs sur les populations de leurs hôtes (Baker et Capinera, 1997 ; Achinelly et Micieli, 2009 ; 2011). Leur production dans les pays en développement est un processus attrayant pour la lutte contre les moustiques vecteurs de maladies (Santamarina et Perez, 1997).

La famille des Mermithidae, comprend 7 différents genres qui sont actuellement identifiés comme parasites des moustiques. Il s'agit des genres Culicimermis, Empidomermis, Hydromermis, Octomyomermis, Perttilimermis, Romanomermis, et Strelkovimermis (Poinar, 2001). Le genre Romanomermis Coman est le plus connu ; il comprend au moins 15 espèces (Tableau 1) dont 14 sont parasites des moustiques (Coman, 1961).

La distribution biogéographique actuelle du genre Romanomermis indique 6 espèces en Chine, 5 en Amérique du Nord, 2 en Inde, 1 au Kazakhstan et 1 en Roumanie. Une des espèces, Romanomermis nielseni Ross et Smith a été identifiée à la fois en Amérique du Nord et en Ukraine (Isaeva et al., 1984). Deux espèces de ce genre ont été étudiées de façon approfondie; il s'agit de Romanomermis culicivorax Ross et Smith et de Romanomermis iyengari (Petersen, 1985).
Les procédures de production en masse sont bien élaborées et il est aujourd'hui possible d'élever facilement de grandes quantités de ces vers (Platzer, 1981).

\section{- Romanomermis iyengari}

Les premières informations sur $R$. iyengari proviennent des observations de Ross (1906) qui a rapporté la présence de nématodes mermithidés dans un échantillon de larves de Culex échantillonné en Inde. Iyengar (1927) a trouvé les juvéniles parasites de nématodes mermithidés dans les larves de 7 différentes espèces d'anophèles. Plus tard, ces nématodes ont été décrits par Welch (1964) comme $R$. iyengari.

Le cycle de développement de $R$. iyengari semble impliquer seulement les stades larvaires des moustiques puisque Gajanana et al. (1978) ont constaté que des pupes de CX. fatigans n'ont pas été infectées par les juvéniles pré-parasitiques. Ainsi, ces auteurs ont conclu que l'infection des larves de $1^{\text {er }}$ et $2^{\text {ème }}$ stades et émergence des nématodes au $4^{\text {ème }}$ stade avec pour résultat la mort de l'hôte était le cours habituel du parasitisme lié à $R$. iyengari. Les parasites juvéniles sont retrouvés au début de l'infection dans toute la cavité du corps de l'hôte infecté mais quelques jours après, ceuxci sont localisés dans l'abdomen des larves d'Anopheles (Iyengar, 1927) alors que chez les Culex, le nématode pénètre dans le thorax pour finir sa croissance.

En général, la température optimale pour l'infection, le développement du parasite, la maturation des post-parasites, la ponte des œufs, le développement embryonnaire, et l'éclosion des œufs pour les deux espèces, $R$. iyengari et $R$. culicivorax, est semblable, et se situe entre $25-30{ }^{\circ} \mathrm{C}$ et 21 $33{ }^{\circ} \mathrm{C}$, respectivement (Platzer, 2007).

Le sable est le milieu dans lequel les nématodes adultes mâles et femelles vivent. La femelle de nématode pond ces œufs dans le sable et les juvéniles infectieux émergent de ces œufs dès que le sable contenant ces œufs est immergé d'eau. La production de 
juvéniles infectieux est optimale lorsque le diamètre des particules de sable est de 1,4 à 2,0 mm (Daim et al., 1987).

De nombreux travaux ont démontré que $R$. iyengari infecte une large gamme d'espèces de moustiques à travers le monde (Pailey et Jayachandran, 1987; Achuthan, 1988 ; Vladimirova et al., 1990 ; Pridantseva et al., 1990 ; Alirzaev et al., 1990). Au Cuba, un pays à climat tropical, des essais en milieu naturel ont démontré que $R$. iyengari décime les populations de différentes espèces de moustiques (Santamarina et al., 1993; Santamarina et Mijares, 1995 ; Santamarina et al., 1996). Au Mexique et au Brésil également, il a été démontré que $R$. iyengari parasite et tue les moustiques vecteurs de maladies $\mathrm{y}$ compris diverses espèces d'anophèles (Santamarina et al., 1999; Santamarina et Bellini, 2000). On connait aujourd'hui plus de 30 espèces de moustiques susceptibles à l'infection de $R$. iyengari (Platzer, 2007).

Différents travaux ont démontré l'efficacité des nématodes dans la lutte contre les moustiques. Au Tadjikistan, Vladimirova et al. (1990) ont démontré que $R$. culicivorax et $R$. iyengari étaient beaucoup plus efficaces contre les Anopheles que les Culex. En Ouzbékistan, Pridantseva et al. (1990) ont obtenu l'infection des anophèles par $R$. iyengari. Des études pour la lutte contre les moustiques conduites en Azerbaijan ont montré que $R$. iyengari infecte à la fois $A n$. sacharovi et Cx. Theileri (Alizaev et al., 1990).

Les résultats de travaux réalisés en milieu naturel à Cuba ont été particulièrement encourageants. Santamarina et al. (1992, 1993) ont démontré que pour la concentration de 1000 nématodes pré-parasitiques de $R$. iyengari par mètre carré de surface, le pourcentage de réduction était de $80-100 \%$ pour les larves d'anophèles et $75-100 \%$ pour les Culex.

Au Mexique, une dose de 2000 à 3000 vers pré-parasitiques de $R$. iyengari par mètre carré de surface a permis d'obtenir un taux d'infection de l'ordre de $85-100 \%$ chez les larves d'An. pseudopunctipennis (Santamarina et al., 1999). Des résultats similaires ont été également obtenus au Brésil où, 12 sites naturels de gîte d'Anopheles ont été traités avec 2.000 nématodes infectieux par mètre carré de surface. Après une semaine, les populations An. albitarsis et An. rondoni ont été réduites de 85-97\% (Santamarina et Bellini, 2000).

Des études ont été effectuées afin de déterminer si $R$. iyengari est inoffensif ou non aux vertébrés. Un grand nombre de préparasitiques infectieux vivants de $R$. iyengari ont été injectés par voie intra-veineuse, intrapéritonéale, intra-dermique, sous-cutanée, et par voies orales, à différents vertébrés. La santé générale des animaux testés (souris adultes, cochons de Guinée, lapins et poules), est restée inchangée. L'histologie des organes comme le foie, la rate, les reins, les poumons et le tractus gastro-intestinal n'a pas montré de lésions liées à une infection par les nématodes. De plus, les poissons (Gambusia affinis) exposés à un grand nombre de préparasitiques infectieux de $R$. iyengari pendant plusieurs jours n'ont pas été affectés de quelque manière que ce soit. Il n'existe donc pas de danger pour la santé et l'environnement avec l'utilisation de $R$. iyengari pour la lutte contre les moustiques (Gajanana et al., 1978).

Des travaux réalisés récemment au Bénin ont prouvé l'efficacité sans faille de $R$. iyengari contre les larves d'Anopheles gambiae. Une dose de 3500 nématodes infectieux par mètre carré a permis de détruire $100 \%$ des larves d'anophèles au bout de 5 jours (Abagli et al., 2012). De plus dans ce même pays, il a été mis au point un système de production à grande échelle de ce ver. La production est basée sur des matériels endogènes accessibles, moins chers et disponibles en Afrique. Ce système de production qui emploie 3 techniciens permet de produire mensuellement des nématodes suffisants pour traiter au moins 75000 mètres carrés de gîtes larvaires (Alavo et al., 2013). 
Tableau 1 : Espèces actuellement décrites de Romanomermis.

\begin{tabular}{lc}
\hline Espèces & Localisation \\
\hline$R$. altaica & Kazakistan \\
$R$. cazanica & Roumanie \\
$R$. chenzhouoensis & Hunan, Chine \\
$R$. communensis & Manitoba, Canada \\
$R$. culicivorax & Louisiane, USA \\
$R$. hermaphrodita & Manitoba, Canada \\
$R$. iyengari & Pondicherry, Inde \\
$R$. jianensis & Jilin, Chine \\
$R$. jingdeercis & Anhui, Chine \\
$R$. kiktoreak & Nunavut, Canada \\
$R$ manjeerensis & Andhra Pradesh, Inde \\
$R$. nielseni & Wyoming, USA \\
$R$. sichuanensis & Sichuan, Chine \\
$R$. wuchangensis & Hubei, Chine \\
$R$. yunanensis & Henan \\
\hline
\end{tabular}

\section{RÉFÉRENCES}

Abagli AZ, Alavo TBC, Platzer EG. 2012. Efficacy of the insect parasitic nematode, Romanomermis iyengari, for malaria vector control in Benin West Africa. Malaria Journal, 11 (1): P5.

Achinelly MF, Micieli MV. 2009. Experimental releases of Strelkovimermis spiculatus (Nematoda: Mermithidae) against three mosquito species in Argentina. Nematology, 11(1): 151-154.

Achinelly MF, Micieli MV. 2011. Optimizing laboratory production of Strelkovimermis spiculatus (Nematoda: Mermithidae) with a discussion of potential release strategies for mosquito biological control. Biological Control, 57: 31-36.

Achuthan C. 1988. Natural parasitic infections in anopheline larvae of Mandya district, Karnataka state. Indian J. Malariol., 25: 59-62.

Alavo TBC, Abagli AZ, Pérez-Pacheco R, Platzer EG. 2013. Successful large scale production of the insect parasitic nematode, Romanomermis iyengari, for sustainable malaria vectors control in Benin, West Africa. The $6^{\text {th }}$ MIM PanAfrican Malaria Conference 6 - 11 October 2013, Durban, South Africa.

Albernaz DAS, Tai MHH, Luz C. 2009. Enhanced ovicidal activity of an oil formulation of the fungus Metarhizium anisopliae on the mosquito Aedes aegypti. Medical and Veterinary Entomology, 23: 141-147.

Alirzaev GU, Pridantseva EP, Vladimirova VV, Alekseev AN. 1990. Prospects for using Romanomermis culicivorax and Romanomermis iyengari Nematoda Mermithida for mosquito control in Azerbaijan USSR. Med Parazitol Parazit Bolezni, 1: 11-15.

Alves SB, Alves LFA, Lopes RB, Pereira RM, Vieira SA. 2002. Potential of some Metarhizium anisopliae isolates for control of Culex quinquefasciatus (Dipt. Culicidae). Journal of Applied Entomology, 126: 504-509.

Andreadis TG, Becnel JJ, White SE. 2003. Infectivity and Pathogenicity of a Novel 
Baculovirus, CuniNPV from Culex nigripalpus (Diptera: Culicidae) for Thirteen Species and Four Genera of Mosquitoes. J. Med. Entomol., 40: 512517.

Armengol G, Hernandez J, Velez JG, Orduz S. 2006. Long-Lasting Effects of a Bacillus thuringiensis Serovar israelensis Experimental Tablet Formulation for Aedes aegypti (Diptera: Culicidae) Control. J. Econ. Entomol., 99(5): 1590 1595.

Asimeng EJ, Mutinga MJ. 1992. Field Evaluation of Tilapia zilli (Gervais) as a Biological Control Agent for Mosquito Control. Biological Control, 2: 317- 320.

Baker GL, Capinera JL. 1997. Nematodes and nematomorphs as control agents of grasshoppers and locusts. Mem. Ent. Soc. Canada, 171: 157-211.

Balkew M, Elhassen I, Ibrahim M, GebreMichael T, Engers H. 2006. Very high DDT-resistant population of Anopheles pharoensis Theobald (Diptera: Culicidae) from Gorgora, northern Ethiopia. Parasite 13(4): 327-329.

Becnel JJ, White SE, Moser BA, Fukuda T, Rotstein MJ, Undeen AH, Cockburn A. 2001. Epizootiology and transmission of a newly discovered baculovirus from the mosquitoes Culex nigripalpus and $C$. quinquefasciatus. J. Gen. Virol., 82: 275282.

Becnel JJ, White SE. 2007. Mosquito pathogenic viruses - the last 20 years. $J$. Am. Mosq. Control Assoc., 23(2): 36-49.

Bhattacharjee I, Aditya G, Chandra G. 2009. Laboratory and field assessment of the potential of larvivorous, air-breathing fishes as predators of culicine mosquitoes. Biological Control., 49: 126-133.

Brown MD, Kay BH, Hendrikz JK. 1991. Evaluation of Australian Mesocyclops (Copepoda: Cyclopidae) for mosquito control. J. Med. Entomol., 28: 618-623.
Büchen-Osmond C. 2003. The universal virus database ICTVdB. Computing Sci. Eng. 5: $16-25$.

Burnham KD, Baldridge RS, Duhrkopf RE, Vodopich DS. 1994. Laboratory study of predation by Solenopsis invicta (Hymenoptera: Formicidae) on eggs of Aedes albopictus (Diptera: Culicidae). J. Med. Entomol., 31: 770-771.

Carson R. 2002. Silent Spring. Mariner Books: USA.

Clark TB, Kellen WR, Fukuda TJ, Lindgren E. 1968. Field and laboratory studies of the pathogenicity of the fungus Beauveria bassiana to three genera of mosquitoes. $J$. Invertebr. Pathol., 11: 1-7.

Coman D. 1961. Mermithidae. In Fauna Republic Populaire Romine (vol 2). Academia Republicii Populare Romine, Fascicula 3. 61 p.

Costa GL, Moraes AML, Oliveira PC. 1998. Pathogenic action of Penicillium species on mosquito vectors of human tropical diseases. Journal of Basic Microbiology, 38: 337-341.

Daim N, Chong NL, Yap HH. 1987. Effect of sand particle size on the production of pre-parasites of Romanomermis iyengari (Nematoda: Mermithidae). Trop. Biomed., 4: 75-77.

De Ley P, Blaxter M. 2002. Systematic position and phylogeny. In The Biology of Nematodes, Lee DL (ed). Taylor and Francis: London; 1-30.

Djogbénou L, Pasteur N, Akogbéto M, Weill M, Chandre F. 2011. Insecticide resistance in the Anopheles gambiae complex in Benin: a nationwide survey. Med. Vet. Entomol., 25: 256-67.

Federici BA. 1985. Viral pathogens of mosquito larvae. Bull. Am. Mosq. Control Assoc., 6: 62-74.

Federici BA. 1995. The future of microbial insecticides as vector control agents. $J$. Am. Mosq. Control. Assoc. 11: 260-268.

Federici BA, Park H-W, Bideshi DK, Wirth MC, Johnson JJ, Sakano Y, Tang M. 
2007. Developing recombinant bacteria for control of mosquito larvae. J. Am. Mosq. Control Assoc., 23(2): 164-175.

Gajanana A, Kazmi SJ, Bheema RUS, Suguna SG, Chandrahas RK. 1978. Studies on a nematode parasite (Romanomermis sp: Mermithidae) of mosquito larvae in Pondicherry. Indian J. Med. Res. 68: 242247.

Geetha I., Manonmani, A.M. 2007. Mosquito pupicidal toxin production by Bacillus subtilis subsp. Subtilis. Biological Control 44: 242-247.

Goettel MS. 1988. Pathogenesis of the hyphomycete Tolypocladium cylindrosporum in the mosquito Aedes aegypti. Journal of Invertebrate Pathology, 51: 259 - 274 .

Govindarajan M, Jebanesan A, Reetha D. 2005. Larvicidal effect of extracellular secondary metabolites of different fungi against the mosquito, Culex quinquefasciatus Say. Tropical Biomedicine, 22: 1-3.

Hati, G. 1965. Aspergillus parasiticus infection in adult mosquitoes. Bulletin of the Calcutta School of Tropical Medicine, 13: $18-19$.

Hurst TP, Kay BH, Brown MD, Ryan PA. 2006. Laboratory evaluation of the effect of alternative prey and vegetation on predation of Culex annulirostris immatures by Australian native fish species. J. Am. Mosq. Control Assoc., 22: 412-417.

Hurst TP, Kay BH, Ryan PA, Brown MD. 2007. Sublethal Effects of Mosquito Larvicides on Swimming Performance of Larvivorous Fish Melanotaenia duboulayi (Atheriniformes: Melanotaeniidae) J. Econ. Entomol., 100(1): 61-65.

Isaeva NM, Dubrovskii Yu-V, Kilochitskii PYa. 1984. Ecology of Romanomermis nielseni (Nematoda, Mermithidae), a parasite of blood-sucking mosquitoes. Vestnik Zoologii, 6: 70-71.
Iyengar MOT. 1927. Parasitic nematodes of Anopheles in Bengal. Trans Far Eastern Assoc Trop Med., 3: 128-135.

de Jager C, Aneck-Hahn NH, Bornman MS, Farias P, Spanò M. 2012. DDT exposure levels and semen quality of young men from a malaria area in South Africa. Malaria Journal, 11(1): P21.

Jousset F, Baquerizo E, Bergoin M. 2000. A new densovirus isolated from the mosquito Culex pipiens (Diptera: culicidae). Virus Res., 67: 11-16.

Kanzok SM, Lorena MJ. 2006. Entomopathogenic fungi as biological insecticides to control malaria. Trends in Parasitology, 22: 49-51.

Kay BH, Cabral CP, Sleigh AC, Brown MD, Ribeiro ZM, Vasconcelos WA. 1992. Laboratory Evaluation of Brazilian Mesocyclops (Copepoda: Cyclopidae) for Mosquito Control. J. Med. Entomol., 29(4): 599-602.

Kellen WR, Clark TB, Lindegren JE. 1963. A possible polyhedrosis in Culex tarsalis Coquillett (Diptera: Culicidae). J. Insect Pathol., 5: 98-103.

Kerry BR, Hominick WM. 2002. Biological control. In The Biology of Nematodes, Lee DL (ed). Taylor and Francis: London; 483-509.

Kerwin JL. 2007. Oomycetes: Lagenidium giganteum. J. Am. Mosq. Control Assoc., 23(2): 50-57.

Kim HC, Kim MS, Yu HS. 1994. Biological control of vector mosquitoes by the use of fish predators, Moroco oxycephalus and Misgurnus anguillicaudatus in the laboratory and semi-field rice paddy. Korean Journal of Entomology, 24: 269284.

Kusumawathie PHD, Wickremasinghe AR, Karunaweera ND, Wijeyaratne MJS. 2006. Larvivorous potential of fish species found in river bed pools below the major dams in Sri Lanka. J. Med. Entomol., 43: 79-82. 
Lacey LA. 2007. Bacillus thuringiensis serovariety Israelensis and bacillus sphaericus for mosquito control. J. Am. Mosq. Control Assoc., 23(2): 133-163.

Ledermann JP, Suchman EL, Black IV WC. Carlson JO. 2004. Infection and Pathogenicity of the Mosquito Densoviruses AeDNV, HeDNV, and APeDNV in Aedes aegypti Mosquitoes (Diptera: Culicidae). J. Econ. Entomol., 97: 1828-1835.

Lee DK, Bhatkar AP, Vinson SB, Olson JK. 1994. Impact of foraging red imported fire ants (Solenopsis invicta) (Hymenoptera: Formicidae) on Psorophora columbiae eggs. J. Am. Mosq. Control Assoc. 10: $163-173$.

Lord JC, Fukuda T. 1988. An ultrastructural study of the invasion of Culex quinquefasciatus larvae by Leptolegna chapmanii (Oomycetes: Saprolegnales). Mycopatholgia 104: 67-73.

Louca V, Lucas MC, Green C, Majambere S, Fillinger U. Lindsay S. 2009. Role of Fish as Predators of Mosquito Larvae on the Floodplain of the Gambia River. J. Med. Entomol., 46(3): 546-556.

Luz C, Tai MHH, Santos AH, Rocha LFN, Albernaz DAS, Silva HHG, 2007. Ovicidal activity of entomopathogenic Hyphomycetes on Aedes aegypti (Diptera: Culicidae) under laboratory conditions. Journal of Medical Entomology, 44: 799-804.

Marti GA, Azpelicueta MM, Tranchida MC, Pelizza SA, Garcia JJ. 2006. Predation efficiency of indigenous larvivorous fish species on Culex pipiens L. larvae (Diptera: Culicidae) in drainage ditches in Argentina. Journal Vector Ecology, 31: 102-106.

Martinez-Ibarra JA, Guillen YG, ArredondoJimenez, J.I. and Rodrigu-Lopez, M.H. 2002. Indigenous fish species for the control of Aedes in water storage tanks in Southern Mexico. BioControl, 47: 481486.
Mogi M. 2007. Insects and other invertebrate predators. J. Am. Mosq. Control Assoc., 23(2): 93-109.

Monnerat R, da Silva SF, Dias DS, Martins ES, Praça LB, Jones GW, Soares CM, de Souza Dias JMC, Berry C. 2004. Screening of Brazilian Bacillus sphaericus strains for high toxicity against Culex quinquefasciatus and Aedes aegypti. JEN., 128(7): 469-473.

Moraes AML, Costa GL, Camargo BMZ, Oliveira RL, Oliveira PC. 2001. The entomopathogenic potential of Aspergillus spp. in mosquitoes vectors of tropical diseases. Journal of Basic Microbiology, 41: 45-49.

Morton RM, Beumer JP, Pollock BR. 1988. Fishes of a subtropical Australian saltmarsh and their predation upon mosquitoes. Environmental Biology of Fishes, 21: 185-194.

Mvoutoulou N. 1992. Larvicidal effects of Penicillium citrinum spores and extracts upon Aedes aegypti. Journal of Invertebrate Pathology, 60: 208 - 209.

Neng W, Shusen W, Guangxin H, Rongman X, Guangkun T, Chen Q. 1987. Control of Aedes aegypti larvae in household containers by Chinese cat fish. Bulletin of the World Health Organization, 65 : 503506.

Ohgushi A, Saitoh H, Wasano N, Ohba M. 2005. A New Insertion Variant, IS231I, Isolated from a Mosquito Specific Strain of Bacillus thuringiensis. Current Microbiology, 51: 95-99.

Oliveira CD, Tadei WP, Abdalla FC. 2009. Occurrence of Apocrine Secretion in the Larval Gut Epithelial Cells of Aedes aegypti L., Anopheles albitarsis LynchArribálzaga and Culex quinquefasciatus Say (Diptera: Culicidae): a Defense Strategy Against Infection by Bacillus sphaericus Neide? Neotropical Entomology, 38(5): 624-631.

OMS. 2011. Rapport 2011 sur le paludisme dans le monde. http://www.who.int/ 
malaria/world_malaria_report_2011/fr/ind ex.html. (Consulté 25 Avril 2012).

Otieno-Ayayo ZN, Zaritsky A, Wirth MC, Manasherob R, Khasdan V, Cahan R, Ben-Dov E. 2008. Variations in the mosquito larvicidal activities of toxins from Bacillus thuringiensis ssp. Israelensis. Environmental Microbiology, 10(9): 2191-2199.

Pailey KP, Jayachandran S. 1987. Factors inhibiting parasitism of mosquito larvae by the mermithid nematode Romanomermis iyengari in a polluted habitat. Indian J. Med. Res. Sect. B, 86: 469-474.

Park H-W, Bideshi DK, Wirth MC, Johnson JJ, Walton WE, Federici BA. 2005. Recombinant larvicidal bacteria with markedly improved efficacy against Culex vectors of West Nile virus. Am. J. Trop. Med. Hyg., 72(6): 732-738.

Petersen JJ. 1985. Nematodes as biological control agents: Part I. Mermithidae. $A d v$ Parasitol, 24: 307-346.

Platzer EG. 1981. Biological control of mosquitoes with mermithids. J. Nematol 13: $257-262$.

Platzer EG, Mullens BA, Shamseldean MM. 2005. Mermithid Nematodes. In Nematodes as Biocontrol Agents, Grewal P, Ehlers R, Shapiro-Ilan D (eds). CABI Publishing: Wallinford, United Kingdom; 411- 418 .

Platzer EG. 2007. Mermithid nematodes. $J$ Am Mosq Control Assoc., 23(2): 58-64.

Poinar GOJr. 2001. Nematoda and Nematomorpha. In Ecology and Classification of North American Freshwater Invertebrates (2nd edn), Thorp JH, Covich AP (eds). Academic Press: San Diego, CA; 255-295.

Poopathi S, Abidha S. 2009. A Medium for the Production of Biopesticides (Bacillus sphaericus and Bacillus thuringiensis subsp. israelensis) in Mosquito Control. J. Econ. Entomol., 102(4): 1423 -1430.
Popiel I, Hominick WM. 1992. Nematodes as biological control agents: Part II. Adv Parasitol., 31: 381-433.

Prabakaran G, Balaraman K. 2006. Development of a cost-effective medium for the large scale production of Bacillus thuringiensis var israelensis. Biological Control, 36: 288-292.

Prabakaran G, Balaraman K, Hoti SL, Manonmani AM. 2007. A cost-effective medium for the large-scale production of Bacillus sphaericus H5a5b (VCRC B42) for mosquito control. Biological Control, 41: 379-383.

Prabakaran G, Hoti SL. 2008. Influence of amino nitrogen in the culture medium enhances the production of $\delta$-endotoxin and biomass of Bacillus thuringiensis var. israelensis for the large-scale production of the mosquito control agent. J. Ind. Microbiol Biotechnol., 35: 961-965.

Prabakaran G, Hoti SL, Paily KP. 2009. Development of cost-effective medium for the large-scale production of a mosquito pupicidal metabolite from Pseudomonas fluorescens Migula. Biological Control, 48: 264-266.

Pridantseva EA, Lebedeva NI, Shcherban ZP, Kadyrova MK. 1990. Assessment of the possibility of using the mermithid Romanomermis iyengari for the control of mosquitoes in Uzbekistan. Meditsinskaya Parazitologiyai Parazitarnye Bolezni, 1: 15-17.

Promdonkoy B, Promdonkoy P, Tanapongpipat S, Luxananil P, Chewawiwat N, Audtho M, Panyim S. 2004. Cloning and Characterization of a Mosquito Larvicidal Toxin Produced during Vegetative Stage of Bacillus sphaericus 2297. Current Microbiology, 49: 84-88.

Quiroz-Martínez, Humberto and RodríguezCastro, Ariadna. 2007. Aquatic insects as predators of mosquito larvae. J Am Mosq Control Assoc., 23(2): 110-117. 
Ramirez-Suero M, Robles-Olvera V, Ramirez-Lepe M. 2005. Spray-Dried Bacillus thuringiensis Serovar israelensis Formulations for Control of Aedes aegypti Larvae. J. Econ. Entomol., 98(5): 1494 1498.

Rees DM. 1934. Notes on mosquito fish in Utah, Gambusia affinis (Baird and Girard). Copeia 1934: 157-159.

Roberts GM. 1995. Salt-marsh Crustaceans, Gammarus duebeni and Palaemonetes varians as Predators of Mosquito Larvae and Their Reaction to Bacillus thuringiensis subsp. israelensis', Biocontrol Science and Technology, 5(3): 379-386.

Ross R. 1906. Notes on the parasites of mosquitoes found in India between 1895 and 1899. J. Hyg., 6: 101-108.

Rout R, Raina V, Suar M, Luethy P. 2011. High prevalence of bacterial sporeformers active against mosquito larvae in temporary monsoon flooded sites in Orissa, India. J. Am. Mosq. Control Assoc., 27(2): 159-161.

Ruiu L, Floris I, Satta A, Ellar DJ. 2007. Toxicity of a Brevibacillus laterosporus strain lacking parasporal crystals against Musca domestica and Aedes aegypti. Biological Control, 43: 136-143.

Russell BM, Kay BH, Shipton W. 2001. Survival of Aedes aegypti (Diptera: Culicidae) eggs in surface and subterranean breeding sites during the Northern Queensland dry season. J. Med. Entomol. 38: 441-445.

Santamarina MA, Garcia AI, Gonzalez BR, Mijares AS, Avila IG, Broche RG. 1992. The ability of the nematode Romanomermis iyengari (Welch, 1964) (Nematoda: Mermithidae) to successfully parasitize mosquito larvae under natural conditions. Rev. Cubana Med. Trop., 44: 92-97.

Santamarina MA, Garcia AI, Gonzalez BR, Mijares AS, Avila IG, Broche RG. 1993. Evaluation of the infective capacity of the parasitic nematode Romanomermis iyengari (Welch, 1964) (Nematoda: Mermithidae) in natural habitats of larval mosquitoes. Rev. Cubana Med. Trop. 45: 128-131.

Santamarina MA, Mijares AS. 1995. Parasitic activity of Romanomermis iyengari in natural breeding sites of mosquito larvae. Misc. Zool., 17: 59-65.

Santamarina MA, Garcia AI, Rivera RJ, Solis MA. 1996. Release of Romanomermis iyengari (Nematoda: Mermithidae) to control Aedes taeniorhynchus (Diptera: Culicidae) in Punta del Este, Isla de la Juventud, Cuba. J. Med. Ent. 33: 680682.

Santamarina MA, Perez PR. 1997. Reduction of mosquito larval densities in natural sites after introduction of Romanomermis culicivorax (Nematoda: Mermithidae) in Cuba. J. Med. Ent., 34: 1-4.

Santamarina MA, Perez PR, Tomas-Martinez SH, Enrique CL, Flores AG. 1999. The Romanomermis iyengari parasite for Anopheles pseudopunctipennis suppression in natural habitats in Oaxaca State, Mexico. Pan. Am. J. Pub. Health, 5: 23-28.

Santamarina MA, Bellini AC. 2000. Mass produced Romanomermis iyengari (Nematoda: Mermithidae) applied to anopheline breeding sites in Boa Vista (Roraima), Brazil. Pan. Am. J. Pub. Health, 7:155-161.

Santos AH, Tai MHH, Rocha LFN, Silva HHG, Luz C. 2009. Dependence of Metarhizium anisopliae on high humidity for ovicidal activity on Aedes aegypti. Biological Control, 50: 37-42.

Sarrafzadeh MH, Guiraud JP, Lagneau C, Gaven B, Carron A, Navarro J-M. 2005. Growth, Sporulation, $\delta$-Endotoxins Synthesis, and Toxicity During Culture of Bacillus thuringiensis H14. Current Microbiology, 51: 75-81.

Scholte EJ, Knols BGJ, Samson RA, Takken W, 2004. Entomopathogenic fungi for 
mosquito control: a review. J. Insect Sci. 4/19: 1-24. (insectscience.org/4.19).

Scholte EJ, Takken W, Knols BGJ, 2007. Infection of adult Aedes aegypti and Ae. albopictus mosquitoes with the entomopathogenic fungus Metarhizium anisopliae. Acta Tropica, 102: 151-158.

Schleier JJ, Sing SE, Peterson RKD. 2007. Regional ecological risk assessment for the introduction of Gambusia affinis (western mosquitofish) into Montana watersheds. Biol Invasions, 10: 12771287.

Schreiber ET. 2007. Toxorhynchites. J. Am Mosq Control Assoc., 23(2): 129-132.

Seye F, Faye O, Ndiaye M, Njie E, Afoutou JM. 2009. Pathogenicity of the fungus, Aspergillus clavatus, isolated from the locust, Oedaleus senegalensis, against larvae of the mosquitoes Aedes aegypti, Anopheles gambiae and Culex quinquefasciatus. Journal of Insect Science, 7: 9-53.

Shapiro AM, Becnel JJ, White SE. 2004. A nucleopolyhedrovirus from Uranotaenia sapphirina (Diptera: Culicidae). J Invertebr Pathol, 86: 96-103.

Silva-Filha MHNL, Chalegre de Melo KD, Anastacio DB, de Oliveira CMF, da Silva SB, Acioli RV, Hibi Sc, de Oliveira DC, Parodi ESM, Filho CAMM, Furtado AF, Regis L. 2008. Culex quinquefasciatus field populations subjected to treatment with Bacillus sphaericus did not display high resistance levels. Biological Control, 44: 227-234.

Siva K, Kadarkarai M. 2011. Laboratory and field evaluation of Metarhizium anisopliae for the control of Dengue vector, Aedes aegypti (Insecta: Diptera: Culicidae). Toxicological \& Environmental Chemistry, 93(6): 11951201.

Soares GG, Riba G, Caudal A, Vincent, JJ. 1985. Comparative studies of eleven isolates of the fungal entomopathogen Tolypocladium cylindrosporum and two isolates of Tolypocladium extinguens. Journal of Invertebrate Pathology, 46: 115-120.

Su T, Mulla MS. 1999. Microbial Agents Bacillus thuringiensis ssp. israelensis and Bacillus sphaericus Suppress Eutrophication, Enhance Water Quality, and Control Mosquitoes in Microcosms. Environ. Entomol., 28(4): 761 - 767.

Suman SM, Kamaraju R, Kumar MP, Aditya PD. 2008. Efficacy of culture filtrates of Metarhizium anisopliae against larvae of Anopheles stephensi and Culex quinquefasciatus. J. Ind. Microbiol. Biotechnol., 35: 1199-1202.

Tranchida MC, Maciá A, Brusa F, Micieli MV, García JJ. 2009. Predation potential of three flatworm species (Platyhelminthes: Turbellaria) on mosquitoes (Diptera: Culicidae). Biological Control, 49: 270-276.

Valero N, Melean E, Maldonado M, Montiel M, Larreal Y, Espina LM. 2006. Larvivorous capacity of the goldfish (Carassius auratus auratus) and the wild guppy (Poecilia reticulata) on larvae of Aedes aegypti in laboratory conditions. Rev. Cient-Fac. Cien., 16: 414-419.

Vladimirova VV, Pridantseva EA, Gafurov AK, Muratova ME. 1990. Testing the mermithids Romanomermis iyengari and $R$. culicivorax for the control of bloodsucking mosquitoes in Tadznik SSR. Meditsinskaya Parazitologiya $i$ Parazitarnye Bolezni., 3: 42-45.

Walton WE. 2007. Larvivorous fish including Gambusia. J. Am. Mosq. Control Assoc., 23(2): 184-220.

Welch HE. 1964. Romanomermis iyengari sp. nov. Nematoda: Mermithidae Braun, 1883. Pilot Register of Zoology, Card No. 4.

Wright MS, Raina AK, Lax AR. 2005. A strain of the fungus Metarhizium anisopliae for controlling subterranean termites. Journal of Economic Entomology, 98: 1451-1458. 
Wu N, Liao G, Li D, Luo Y, Zhong G. 1991. The advantages of mosquito biocontrol by stocking edible fish in rice paddies. Southeast Asian Journal of Tropical Medicine and Public Health, 22: 436442.

Xudong X, Renqiu K, Yuxiang H. 1993. High larvicidal activity of intact recombinant cyanobacterium Anabaena sp. PCC 7120 expressing Gene 51 and Gene 42 of Bacillus sphaericus sp. 2297. FEMS Microbiology Letters, 107: 247-250.

Yadav RS, Padhan K, Sharma VP. 1992. Fishes of district Sundargarh, Orissa, with special reference to their potential in mosquito control. Indian J. Malariol., 29: 225-233.

Yu HS. 1986. Biological control of malaria vector, Anopheles sinensis Wiedemann by the release of larvivorous fish, Aplocheilus latipes in simulated rice paddies in Korea. Korean Journal of Entomology, 16: 93-99.

Yu HS, Kim HC. 1993. Integrated control of encephalitis vector (Culex tritaeniorhynchus) with native fishes (Aplocheilus latipes and Aphyocypris chinensis) and Bacillus thuringiensis (H14) in marshes in Jindo Island of Korea. Korean Journal of Entomology, 23: 221230.

Zahiri NS, Su T, Mulla MS. 2002. Strategies for the Management of Resistance in Mosquitoes to the Microbial Control Agent Bacillus sphaericus. J. Med. Entomol., 39(3): 513 - 520. 